\title{
A Reaction-Diffusion System with Nonlinear Nonlocal Boundary Conditions
}

\author{
Alexander Gladkov ${ }^{1}$ and Alexandr Nikitin ${ }^{2}$ \\ ${ }^{1}$ Department of Mechanics and Mathematics, Belarusian State University, Nezavisimosti Avenue 4, 220030 Minsk, Belarus \\ ${ }^{2}$ Department of Mathematics, Vitebsk State University, Moskovsky Avenue 33, 210038 Vitebsk, Belarus
}

Correspondence should be addressed to Alexander Gladkov; gladkoval@mail.ru

Received 24 October 2013; Accepted 23 December 2013; Published 20 February 2014

Academic Editor: Roberto Natalini

Copyright (C) 2014 A. Gladkov and A. Nikitin. This is an open access article distributed under the Creative Commons Attribution License, which permits unrestricted use, distribution, and reproduction in any medium, provided the original work is properly cited.

We consider initial boundary value problem for a reaction-diffusion system with nonlinear and nonlocal boundary conditions and nonnegative initial data. We prove local existence, uniqueness, and nonuniqueness of solutions.

\section{Introduction}

In this paper we consider the following semilinear reactiondiffusion system with nonlinear nonlocal boundary conditions:

$$
\begin{array}{r}
u_{t}=\Delta u+c_{1}(x, t) v^{p}, \quad v_{t}=\Delta v+c_{2}(x, t) u^{q}, \\
x \in \Omega, \quad 0<t<T, \\
u(x, t)=\int_{\Omega} k_{1}(x, y, t) u^{m}(y, t) d y, \\
x \in \partial \Omega, \quad 0<t<T, \\
v(x, t)=\int_{\Omega} k_{2}(x, y, t) v^{n}(y, t) d y, \\
x \in \partial \Omega, \quad 0<t<T, \\
u(x, 0)=u_{0}(x), \quad v(x, 0)=v_{0}(x), \quad x \in \Omega,
\end{array}
$$

where $p, q, m, n>0, \Omega$ is a bounded domain in $\mathbb{R}^{N}$ for $N \geq 1$ with smooth boundary $\partial \Omega, T>0$. Here, $c_{1}(x, t), c_{2}(x, t)$ are nonnegative Hölder continuous functions defined for $x \in \bar{\Omega}$, and $t \in[0, T]$ and $k_{1}(x, y, t), k_{2}(x, y, t)$ are nonnegative continuous functions defined for $x \in \partial \Omega, y \in \bar{\Omega}$, and $t \in[0, T]$. The initial data $u_{0}(x), v_{0}(x)$ are nonnegative continuous functions satisfying the boundary conditions at $t=0$.
In the past several decades, many physical phenomena have been formulated into nonlocal mathematical models. Initial boundary value problem for semilinear reactiondiffusion equations and systems with nonlocal boundary conditions has been analyzed by many authors (see, e.g., [112] and the references therein). Local and global existence, comparison principle, and various qualitative properties have been discussed.

We note that for $\max (p, q, m, n)<1$ the nonlinearities in (1) are non-Lipschitzian. The problem of uniqueness and nonuniqueness for different nonlinear parabolic equations and systems with non-Lipschitzian data has been addressed by several authors. See, for example, [13-16] for equations and [17-21] for systems. In particular, the authors of [16] have investigated the uniqueness of solution for a problem (1) with a single equation.

In [7] the authors have considered a problem (1) with $c_{i}(x, t)=1$ and $k_{i}(x, y, t)=k_{i}(x, y), i=1,2$. They have proved a comparison principal and investigated the blowup properties of the positive solutions. The aim of this paper is to study the uniqueness of the nonnegative solution of the problem (1) for any $p, q, m, n>0$.

The plan of this paper is as follows. In the next section we prove a comparison principle; an existence theorem of a local solution is given in Section 3; uniqueness of solutions with nontrivial initial data, uniqueness of solution with trivial initial datum for $\min (p, q, m, n) \geq 1$; nonuniqueness of 
solution with trivial initial datum for $\min (p q, m, n)<1$ are proved in Section 4.

\section{Comparison Principle}

Let us introduce the definitions of a subsolution and a supersolution. For the remainder of this paper we denote $Q_{T}=\Omega \times(0, T), S_{T}=\partial \Omega \times(0, T)$.

Definition 1. A pair of nonnegative functions $(u, v) \in$ $C^{2,1}\left(Q_{T}\right) \bigcap C\left(\bar{Q}_{T}\right)$ is called a subsolution of problem (1) in $Q_{T}$ if

$$
\begin{gathered}
u_{t} \leq \Delta u+c_{1}(x, t) v^{p}, \quad v_{t} \leq \Delta v+c_{2}(x, t) u^{q}, \\
x \in \Omega, \quad 0<t<T, \\
u(x, t) \leq \int_{\Omega} k_{1}(x, y, t) u^{m}(y, t) d y, \\
x \in \partial \Omega, \quad 0<t<T, \\
v(x, t) \leq \int_{\Omega} k_{2}(x, y, t) v^{n}(y, t) d y, \\
x \in \partial \Omega, \quad 0<t<T, \\
u(x, 0) \leq u_{0}(x), \quad v(x, 0) \leq v_{0}(x), \quad x \in \Omega,
\end{gathered}
$$

and a pair of nonnegative functions $(u, v) \in C^{2,1}\left(Q_{T}\right) \bigcap$ $C\left(\bar{Q}_{T}\right)$ is called a supersolution of problem (1) in $Q_{T}$ if the reversed inequalities hold in (2).

Definition 2. A pair of functions $(u, v)$ is called a solution of (1) in $Q_{T}$ if it is both a subsolution and a supersolution of problem (1) in $Q_{T}$.

Definition 3. We say that solution $(u, v)$ of $(1)$ is positive in $Q_{T}$ if $u>0$ and $v>0$ in $Q_{T}$.

To establish the uniqueness results we need a comparison principle. We prove it in a different way, not as in the work of [7].

Theorem 4. Let $(\bar{u}, \bar{v})$ and $(\underline{u}, \underline{v})$ be a nonnegative supersolution and a nonnegative subsolution of problem (1) in $Q_{T}$, respectively. Suppose that $\bar{u}>0$ and $\bar{v}>0$ or $u>0$ and $\underline{v}>0$ in $\bar{Q}_{T}$ if $\min (p, q, m, n)<1$. If $\bar{u}(x, 0) \geq \underline{u}(x, 0)$ and $\bar{v}(x, 0) \geq \underline{v}(x, 0)$ for $x \in \bar{\Omega}$, then $\bar{u} \geq \underline{u}$ and $\bar{v} \geq \underline{v}$ in $\bar{Q}_{T}$.

Proof. Let $\xi(x, t) \in C^{2,1}\left(\bar{Q}_{T}\right)$ be a nonnegative function such that $\left.\xi\right|_{S_{T}}=0$. Then $\partial \xi /\left.\partial \nu\right|_{S_{T}} \leq 0$, where $\nu$ is the unit outward normal to the lateral boundary of $Q_{T}$. By the definition of a subsolution we have

$$
\underline{u}_{t} \leq \Delta \underline{u}+c_{1}(x, t) \underline{v}^{p}, \quad x \in \Omega, 0<t<T .
$$

If we multiply (3) by $\xi$ and then integrate over $Q_{t}$ for $0<t<$ $T$, we get

$$
\begin{aligned}
\int_{\Omega} \underline{u}(x, t) \xi(x, t) d x \\
\leq \int_{\Omega} \underline{u}(x, 0) \xi(x, 0) d x \\
\quad+\int_{0}^{t} \int_{\Omega}\left(\underline{u}_{\tau}+\underline{u} \Delta \xi+c_{1}(x, \tau) \underline{v}^{p} \xi\right) d x d \tau \\
\quad-\int_{0}^{t} \int_{\partial \Omega} \frac{\partial \xi}{\partial \nu}\left(\int_{\Omega} k_{1}(x, y, \tau) \underline{u}^{m}(y, \tau) d y\right) d s d \tau .
\end{aligned}
$$

On the other hand, the supersolution $\bar{u}$ satisfies (4) with reversed inequality. Set $w(x, t)=\underline{u}(x, t)-\bar{u}(x, t), z(x, t)=$ $\underline{v}(x, t)-\bar{v}(x, t)$. Then we have

$$
\begin{aligned}
& \int_{\Omega} w(x, t) \xi(x, t) d x \\
& \leq \int_{\Omega} w(x, 0) \xi(x, 0) d x+\int_{0}^{t} \int_{\Omega} w\left(\xi_{\tau}+\Delta \xi\right) d x d \tau \\
& \quad-\int_{0}^{t} \int_{\partial \Omega} \frac{\partial \xi}{\partial \nu} \\
& \quad \times\left(\int_{\Omega} k_{1}(x, y, \tau) m \Theta_{2}^{m-1}(y, \tau) w(y, \tau) d y\right) d s d \tau \\
& \quad+\int_{0}^{t} \int_{\Omega} z(x, t) p c_{1}(x, t) \Theta_{1}^{p-1} \xi d x d \tau,
\end{aligned}
$$

where $\Theta_{1}, \Theta_{2}$ are nonnegative continuous functions if $\min (p, q, m, n) \geq 1$ and positive continuous functions if $\min (p, q, m, n)<1$ in $\bar{Q}_{T}$ which satisfy the following equalities:

$$
\begin{aligned}
& \underline{v}^{p}-\bar{v}^{p}=p \Theta_{1}^{p-1}(\underline{v}-\bar{v}), \\
& \underline{u}^{m}-\bar{u}^{m}=m \Theta_{2}^{m-1}(\underline{u}-\bar{u}) .
\end{aligned}
$$

Obviously there exists a positive constant $M_{1}$ such that $\Theta_{1}^{p-1} \leq M_{1}, \Theta_{2}^{m-1} \leq M_{1}$ in $\bar{Q}_{T}$. Since $c_{1}(x, t), k_{1}(x, y, t)$ are nonnegative and continuous functions, then there exists a constant $M_{2}>0$ such that $0 \leq c_{1}(x, t) \leq M_{2}, 0 \leq k_{1}(x, y, t) \leq$ $M_{2}$ in $\bar{Q}_{T}$ and $\partial \Omega \times \bar{Q}_{T}$, respectively.

Consider the following backward problem in $Q_{t}$ :

$$
\begin{gathered}
\xi_{\tau}+\Delta \xi=0, \quad(x, \tau) \in Q_{t}, \\
\xi=0, \quad(x, \tau) \in S_{t}, \\
\xi(x, t)=\psi(x), \quad x \in \Omega,
\end{gathered}
$$

where $\psi(x) \in C_{0}^{\infty}(\Omega), 0 \leq \psi(x) \leq 1$. By the maximum principle for the heat equation $0 \leq \xi \leq 1$, it is easy to show that $-M_{3} \leq \partial \xi / \partial v \leq 0$ on $S_{t}$ for some $M_{3} \geq 0$.

Let $s_{+}=\max (s, 0)$. Then from (5), we get

$$
\begin{aligned}
\int_{\Omega} w(x, t) \psi(x) d x \leq & K_{1} \int_{0}^{t} \int_{\Omega} z_{+}(x, t) d x d t \\
& +K_{2} \int_{0}^{t} \int_{\Omega} w_{+}(y, \tau) d y d \tau,
\end{aligned}
$$


where $K_{1}=p M_{1} M_{2}, K_{2}=m M_{1} M_{2} M_{3}|\partial \Omega|$, and $|\partial \Omega|$ is the Lebesgue measure of $\partial \Omega$. Consider the sequence of functions $\left\{\phi_{n}(x)\right\}, \phi_{n}(x) \in C_{0}^{\infty}(\bar{\Omega}), 0 \leq \phi_{n} \leq 1$, converging in $L^{1}(\Omega)$ to $\phi(x)$, defined as follows:

$$
\begin{array}{ll}
\phi(x)=1, & \text { if } w(x, t)>0, \\
\phi(x)=0, & \text { if } w(x, t) \leq 0 .
\end{array}
$$

Replacing $\psi(x)$ by $\phi_{n}(x)$ in (8) and passing to the limit as $n \rightarrow$ $\infty$, we have

$$
\begin{aligned}
\int_{\Omega} w_{+}(x, t) d x \leq & K_{1} \int_{0}^{t} \int_{\Omega} z_{+}(x, t) d x d t \\
& +K_{2} \int_{0}^{t} \int_{\Omega} w_{+}(y, \tau) d y d \tau .
\end{aligned}
$$

Using a similar argument for the inequality $\underline{v}_{t} \leq \Delta \underline{v}+$ $c_{2}(x, t) \underline{u}^{q}, x \in \Omega, 0<t<T$, we get

$$
\begin{aligned}
\int_{\Omega} z_{+}(x, t) d x \leq & K_{3} \int_{0}^{t} \int_{\Omega} w_{+}(x, t) d x d t \\
& +K_{4} \int_{0}^{t} \int_{\Omega} z_{+}(y, \tau) d y d \tau
\end{aligned}
$$

where $K_{3}, K_{4}$ are positive constants. Adding (10) and (11), we have

$$
\begin{aligned}
& \int_{\Omega}\left(w_{+}(x, t)+z_{+}(x, t)\right) d x \\
& \leq K_{5} \int_{0}^{t} \int_{\Omega}\left(w_{+}(x, t)+z_{+}(x, t)\right) d x d t
\end{aligned}
$$

where $K_{5}=\max \left(K_{1}+K_{4}, K_{2}+K_{3}\right)$.

Applying now Gronwall's inequality, we conclude that

$$
\int_{\Omega}\left(w_{+}(x, t)+z_{+}(x, t)\right) d x \leq 0
$$

Since $w_{+}+z_{+} \geq 0$, then $\underline{u}(x, t)-\bar{u}(x, t) \leq 0, \underline{v}(x, t)-\bar{v}(x, t) \leq$ 0 .

\section{Local Existence}

Let $\left\{\varepsilon_{l}\right\}$ be decreasing to 0 sequence such that $0<\varepsilon_{l}<1$. For $\varepsilon=\varepsilon_{l}$ let $u_{0 \varepsilon}(x), v_{0 \varepsilon}(x)$ be the functions with the following properties: $u_{0 \varepsilon}(x), v_{0 \varepsilon}(x) \in C(\bar{\Omega}), u_{0 \varepsilon}(x) \geq \varepsilon, v_{0 \varepsilon}(x) \geq \varepsilon$; $u_{0 \varepsilon_{i}}(x) \geq u_{0 \varepsilon_{j}}(x), v_{0 \varepsilon_{i}}(x) \geq v_{0 \varepsilon_{j}}(x)$ for $i<j ; u_{0 \varepsilon}(x) \rightarrow$ $u_{0}(x), v_{0 \varepsilon}(x) \rightarrow v_{0}(x)$ as $\varepsilon \rightarrow 0 ; u_{0 \varepsilon}(x)=\int_{\Omega} k_{1}(x, y$, $0) u_{0 \varepsilon}^{m}(y) d y+\varepsilon, \quad v_{0 \varepsilon}(x)=\int_{\Omega} k_{2}(x, y, 0) v_{0 \varepsilon}^{n}(y) d y+\varepsilon$ for $x \in \partial \Omega$.
Due to the nonlinearities in (1), the Lipschitz condition is not satisfied if $\min (p, q, m, n)<1$, and thus we need to consider the following auxiliary problem:

$$
\begin{gathered}
u_{t}=\Delta u+c_{1}(x, t) v^{p}, \quad v_{t}=\Delta v+c_{2}(x, t) u^{q}, \\
x \in \Omega, \quad 0<t<T, \\
u(x, t)=\int_{\Omega} k_{1}(x, y, t) u^{m}(y, t) d y+\varepsilon, \\
x \in \partial \Omega, \quad 0<t<T, \\
v(x, t)=\int_{\Omega} k_{2}(x, y, t) v^{n}(y, t) d y+\varepsilon, \\
x \in \partial \Omega, \quad 0<t<T, \\
u(x, 0)=u_{0 \varepsilon}(x), \quad x(x, 0)=v_{0 \varepsilon}(x), \quad x \in \Omega .
\end{gathered}
$$

Theorem 5. For small values of $T$, (14) has a unique solution in $Q_{T}$.

Proof. We start the proof with the construction of a supersolution of (14). Let $\max \left(\sup _{\Omega} u_{0 \varepsilon}(x), \sup _{\Omega} v_{0 \varepsilon}(x)\right) \leq C$, $C>0$. Denote $M=\max \left(\sup _{Q_{T}} c_{1}(x, t), \sup _{Q_{T}} c_{2}(x, t)\right.$, $\left.\sup _{\partial \Omega \times Q_{T}} k_{1}(x, y, t), \sup _{\partial \Omega \times Q_{T}} k_{2}(x, y, t)\right)$. Introduce an auxiliary function $\varphi(x)$ with the following properties:

$$
\begin{gathered}
\varphi(x) \in C^{2}(\bar{\Omega}), \\
\inf _{\Omega} \varphi(x) \geq 1,
\end{gathered}
$$

$$
\inf _{\partial \Omega} \varphi(x) \geq \bar{M} \int_{\Omega} \max \left(\varphi^{m}(y), \varphi^{n}(y)\right) d y+C^{-1},
$$

where $\bar{M}=M \max \left(C^{m-1}, C^{n-1}\right) \max (1, \exp (m-1), \exp (n-$ $1)$ ). Let $\alpha, \beta$ be positive constants such that $\alpha q-\beta=\beta p-\alpha$ and

$$
\begin{aligned}
& \alpha \geq \sup _{\Omega}\left(\frac{\Delta \varphi}{\varphi}+M C^{p-1} \exp (1) \varphi^{p-1}\right), \\
& \beta \geq \sup _{\Omega}\left(\frac{\Delta \varphi}{\varphi}+M C^{q-1} \exp (1) \varphi^{q-1}\right) .
\end{aligned}
$$

Note that $\alpha q-\beta>0$ for $p q>1$ and $\alpha q-\beta \leq 0$ for $p q \leq 1$. Obviously the pair of functions $(\varepsilon, \varepsilon)$ is a subsolution of problem (14). We show that

$$
\begin{aligned}
& f(x, t)=C \exp (\alpha t) \varphi(x), \\
& g(x, t)=C \exp (\beta t) \varphi(x)
\end{aligned}
$$


is a supersolution of $(14)$ in $Q_{T}$ for $T \leq \min (1 / \alpha, 1 / \beta, 1 /(\alpha q-$ $\beta)$ ) if $p q>1$ and $T \leq \min (1 / \alpha, 1 / \beta)$ if $p q \leq 1$. We have

$$
\begin{aligned}
& f_{t}(x, t)-\Delta f(x, t)-c_{1}(x, t) g^{p}(x, t) \\
&= \alpha C \exp (\alpha t) \varphi(x)-\Delta \varphi C \exp (\alpha t) \\
&-c_{1}(x, t) C^{p} \exp (\beta p t) \varphi^{p}(x) \\
& \geq f(x, t)\left(\alpha-\frac{\Delta \varphi(x)}{\varphi(x)}-M C^{p-1} \exp ((\beta p-\alpha) t) \varphi^{p-1}\right) \\
& \geq 0,
\end{aligned}
$$

for $(x, t) \in Q_{T}$. On the other hand, we get that

$$
\begin{aligned}
C \exp (\alpha t) \varphi(x) & \geq C^{m} \exp (\alpha m t) M \int_{\Omega} \varphi^{m}(y) d y+1 \\
& \geq \int_{\Omega} k_{1}(x, y, t) f^{m}(y, t) d y+\varepsilon,
\end{aligned}
$$

for $(x, t) \in S_{T}$. Similarly, we can show that

$$
\begin{gathered}
g_{t}(x, t)-\Delta g(x, t)-c_{2}(x, t) f^{q}(x, t) \geq 0, \quad \text { for }(x, t) \in Q_{T}, \\
g(x, t) \geq \int_{\Omega} k_{2}(x, y, t) g^{n}(y, t) d y+\varepsilon, \quad \text { for }(x, t) \in S_{T} .
\end{gathered}
$$

To prove the existence of a solution of the problem (14) we introduce the set

$$
\begin{gathered}
B=\left\{\left(h_{1}, h_{2}\right) \in C\left(\bar{Q}_{T}\right) \times C\left(\bar{Q}_{T}\right): \varepsilon \leq h_{1} \leq f,\right. \\
\varepsilon \leq h_{2} \leq g, h_{1}(x, 0)=u_{0 \varepsilon}(x), \\
\left.h_{2}(x, 0)=v_{0 \varepsilon}(x)\right\} .
\end{gathered}
$$

Clearly $B$ is a nonempty convex subset of $C\left(\bar{Q}_{T}\right) \times C\left(\bar{Q}_{T}\right)$.

Consider the following problem:

$$
\begin{gathered}
u_{t}=\Delta u+c_{1}(x, t) v^{p}, \quad v_{t}=\Delta v+c_{2}(x, t) u^{q}, \\
x \in \Omega, \quad 0<t<T, \\
u(x, t)=\int_{\Omega} k_{1}(x, y, t) s_{1}^{m}(y, t) d y+\varepsilon, \\
x \in \partial \Omega, \quad 0<t<T, \\
v(x, t)=\int_{\Omega} k_{2}(x, y, t) s_{2}^{n}(y, t) d y+\varepsilon, \\
x \in \partial \Omega, \quad 0<t<T, \\
u(x, 0)=u_{0 \varepsilon}(x), \quad v(x, 0)=v_{0 \varepsilon}(x), \quad x \in \Omega,
\end{gathered}
$$

where $\left(s_{1}, s_{2}\right) \in B$. Problem (22) has a nontrivial positive solution. Let us call $A\left(s_{1}, s_{2}\right)=(u, v)$. In order to show that $A$ has a fixed point in $B$ we verify that $A$ is a continuous mapping from $B$ into itself such that $A B$ is relatively compact. Thanks to the comparison principle for (22) we have that $A$ maps $B$ into itself.

Let $G(x, y ; t)$ denote the Green's function for the heat equation given by

$$
u_{t}-\Delta u=0, \quad x \in \Omega, \quad t>0
$$

with zero boundary condition. Then $(u, v)$ is a solution of (22) if and only if

$$
\begin{aligned}
u(x, t)= & \int_{\Omega} G(x, y ; t) u_{0 \varepsilon}(y) d y \\
& +\int_{0}^{t} \int_{\Omega} G(x, y ; t-\tau) c_{1}(y, \tau) v^{p}(y, \tau) d y d \tau \\
& -\int_{0}^{t} \int_{\partial \Omega} \frac{\partial G}{\partial \nu}(x, \xi ; t-\tau) \\
& \times\left(\int_{\Omega} k_{1}(\xi, y, \tau) s_{1}^{m}(y, \tau) d y+\varepsilon\right) d \xi d \tau, \\
v(x, t)= & \int_{\Omega} G(x, y ; t) v_{0 \varepsilon}(y) d y \\
& +\int_{0}^{t} \int_{\Omega} G(x, y ; t-\tau) c_{2}(y, \tau) u^{q}(y, \tau) d y d \tau \\
& -\int_{0}^{t} \int_{\partial \Omega} \frac{\partial G}{\partial \nu}(x, \xi ; t-\tau) \\
& \times\left(\int_{\Omega} k_{2}(\xi, y, \tau) s_{2}^{n}(y, \tau) d y+\varepsilon\right) d \xi d \tau .
\end{aligned}
$$

We claim that $A$ is continuous. In fact, let $\left\{\left(s_{1 k}, s_{2 k}\right)\right\}$ be a sequence in $B$ converging to $\left(s_{1}, s_{2}\right) \in B$ in $C\left(\bar{Q}_{T}\right) \times C\left(\bar{Q}_{T}\right)$. Denote $\left(u_{k}, v_{k}\right)=A\left(s_{1 k}, s_{2 k}\right)$. Then we see that

$$
\begin{aligned}
& \left|u-u_{k}\right|+\left|v-v_{k}\right| \\
& \leq \int_{0}^{t} \int_{\Omega} G(x, y ; t-\tau) c_{1}(y, \tau)\left|v^{p}-v_{k}^{p}\right| d y d \tau \\
& \quad+\int_{0}^{t} \int_{\Omega} G(x, y ; t-\tau) c_{2}(y, \tau)\left|u^{q}-u_{k}^{q}\right| d y d \tau \\
& \quad-\sup _{\mathrm{Q}_{T}}\left|s_{1}^{m}-s_{1 k}^{m}\right| \int_{0}^{t} \int_{\partial \Omega} \frac{\partial G}{\partial \nu}(x, \xi ; t-\tau) \\
& \quad \times \int_{\Omega} k_{1}(\xi, y, \tau) d y d \xi d \tau \\
& \quad-\sup _{\mathrm{Q}_{T}}\left|s_{2}^{n}-s_{2 k}^{n}\right| \int_{0}^{t} \int_{\partial \Omega} \frac{\partial G}{\partial \nu}(x, \xi ; t-\tau) \\
& \quad \times \int_{\Omega} k_{2}(\xi, y, \tau) d y d \xi d \tau
\end{aligned}
$$




$$
\begin{aligned}
& \leq r\left(\sup _{Q_{T}}\left|u-u_{k}\right|+\sup _{Q_{T}}\left|v-v_{k}\right|\right) \\
& -\sup _{Q_{T}}\left|s_{1}^{m}-s_{1 k}^{m}\right| \int_{0}^{t} \int_{\partial \Omega} \frac{\partial G}{\partial \nu}(x, \xi ; t-\tau) \\
& \quad \times \int_{\Omega} k_{1}(\xi, y, \tau) d y d \xi d \tau \\
& -\sup _{Q_{T}}\left|s_{2}^{n}-s_{2 k}^{n}\right| \int_{0}^{t} \int_{\partial \Omega} \frac{\partial G}{\partial \nu}(x, \xi ; t-\tau) \\
& \quad \times \int_{\Omega} k_{2}(\xi, y, \tau) d y d \xi d \tau,
\end{aligned}
$$

where $r=\max \left(\Theta_{1}, \Theta_{2}\right)$ and

$$
\begin{aligned}
\Theta_{1}= & p \max \left(\varepsilon^{p-1}, \operatorname{suph}_{\mathrm{Q}_{T}}^{p-1}(x, t)\right) \\
& \times \sup _{\mathrm{Q}_{T}} \int_{0}^{t} \int_{\Omega} G(x, y ; t-\tau) c_{1}(y, \tau) d y d \tau, \\
\Theta_{2}= & q \max \left(\varepsilon^{q-1}, \operatorname{suph}_{2}^{q-1}(x, t)\right) \\
& \times \sup _{\mathrm{Q}_{T}} \int_{0}^{t} \int_{\Omega} G(x, y ; t-\tau) c_{2}(y, \tau) d y d \tau .
\end{aligned}
$$

Choosing $T$ so small that $r<1$, we conclude that $\left(u_{k}, v_{k}\right) \rightarrow$ $(u, v)$ in $C\left(\bar{Q}_{T}\right) \times C\left(\bar{Q}_{T}\right)$ as $k \rightarrow \infty$. The equicontinuity of $A B$ follows from (24), (25) and the properties of the Green's function (see, e.g. [22]). The Ascoli-Arzela theorem guarantees the relative compactness of $A B$. Thus, we are able to apply the Schauder-Tychonoff fixed point theorem and conclude that $A$ has a fixed point in $B$ if $T$ is small. Since $(u, v)$ is a fixed point of $A$, it is a solution of (14). Uniqueness of solution follows from a comparison principle for (14) which can be proved in a similar way as in the previous section.

Using Theorem 5, we can prove the following local existence theorem of a solution of problem (1).

Theorem 6. For small values of $T$ (1) has a maximal solution in $Q_{T}$.

Proof. Let $\varepsilon_{2}>\varepsilon_{1}$. It is easy to show that $\left(u_{\varepsilon_{2}}(x, t), v_{\varepsilon_{2}}(x, t)\right)$ is a supersolution of the problem (14) with $\varepsilon=\varepsilon_{1}$. Then $u_{\varepsilon_{1}}(x, t)$ $\leq u_{\varepsilon_{2}}(x, t), v_{\varepsilon_{1}}(x, t) \leq v_{\varepsilon_{2}}(x, t)$. Using these inequalities and the continuation principle of solutions we deduce that the existence time of $\left(u_{\varepsilon}(x, t), v_{\varepsilon}(x, t)\right)$ does not decrease as $\varepsilon \rightarrow$ 0 . Let $\varepsilon \rightarrow 0$, then

$$
\begin{gathered}
u_{\max }(x, t)=\lim _{\varepsilon \rightarrow 0} u_{\varepsilon}(x, t) \geq 0, \\
v_{\max }(x, t)=\lim _{\varepsilon \rightarrow 0} v_{\varepsilon}(x, t) \geq 0,
\end{gathered}
$$

and $\left(u_{\max }(x, t), v_{\max }(x, t)\right)$ exist in $Q_{T}$ for some $T>0$.
Moreover, by dominated convergence theorem, $\left(u_{\max }(x, t), v_{\max }(x, t)\right)$ satisfies the following equations:

$$
\begin{aligned}
u_{\max }(x, t)= & \int_{\Omega} G(x, y ; t) u_{0}(y) d y \\
& +\int_{0}^{t} \int_{\Omega} G(x, y ; t-\tau) c_{1}(y, \tau) \\
& \times v_{\max }^{p}(y, \tau) d y d \tau \\
& -\int_{0}^{t} \int_{\partial \Omega} \frac{\partial G}{\partial \nu}(x, \xi ; t-\tau) \\
& \times\left(\int_{\Omega} k_{1}(\xi, y, \tau) u_{\max }^{m}(y, \tau) d y\right) d \xi d \tau, \\
v_{\max }(x, t)= & \int_{\Omega} G(x, y ; t) v_{0}(y) d y \\
& +\int_{0}^{t} \int_{\Omega} G(x, y ; t-\tau) c_{2}(y, \tau) \\
& \times u_{\max }^{q}(y, \tau) d y d \tau \\
& -\int_{0}^{t} \int_{\partial \Omega} \frac{\partial G}{\partial \nu}(x, \xi ; t-\tau) \\
& \times\left(\int_{\Omega} k_{2}(\xi, y, \tau) v_{\max }^{n}(y, \tau) d y\right) d \xi d \tau .
\end{aligned}
$$

The interior regularity of $\left(u_{\max }(x, t), v_{\max }(x, t)\right)$ follows from the continuity of $\left(u_{\max }(x, t), v_{\max }(x, t)\right)$ in $Q_{T}$ and the properties of the Green's function. Obviously $\left(u_{\max }(x, t), v_{\max }(x, t)\right)$ satisfies (1). Let $\left(y_{1}(x, t), y_{2}(x, t)\right)$ be any other solution of $(1)$. Then by the comparison principle $u_{\varepsilon}(x, t) \geq y_{1}(x, t), v_{\varepsilon}(x, t)$ $\geq y_{2}(x, t)$. Taking $\varepsilon \rightarrow 0$, we conclude that $u_{\max } \geq y_{1}(x, t)$, $v_{\max }(x, t) \geq y_{2}(x, t)$.

To prove the positiveness of nontrivial solutions we need the following definition.

Definition 7. We say that a function $g(x, t)$ has the property $(N)$ if there exist $x_{k} \in \Omega$ and $t_{k}>0, k \in \mathbb{N}$ such that $g\left(x_{k}\right.$, $\left.t_{k}\right)>0, k \in \mathbb{N}$, and $t_{k} \rightarrow 0$ as $k \rightarrow \infty$.

Remark 8. Note that if a nonnegative function $g(x, t)$ has no the property $(N)$ then $g(x, t) \equiv 0$ in $Q_{\tau}$ for some $\tau>0$.

Theorem 9. Let either $u_{0}(x)$ or $v_{0}(x)$ be a nontrivial function in $\Omega$. Supposing that $k_{1}(x, \cdot, t)$ and $k_{2}(x, \cdot, t)$ are nontrivial functions for any $x \in \partial \Omega$ and $t \in(0, T), c_{1}(x, t)$ has the property $(N)$ if $u_{0}(x) \equiv 0$, and $c_{2}(x, t)$ has the property $(N)$ if $v_{0}(x) \equiv 0$. Let $(u(x, t), v(x, t))$ be a supersolution of (1) in $Q_{T}$. Then $(u(x, t), v(x, t))$ is positive in $\bar{Q}_{T}$ for $0<t<T$.

Proof. Suppose for definiteness that $u_{0}(x)$ is a nontrivial function. We show at first that $u(x, t)>0$ in $\bar{Q}_{T}$ for $0<t<T$. We have

$$
u_{t}-\Delta u \geq c_{1}(x, t) v^{p} \geq 0
$$

then by strong maximum principle a minimum of $u(x, t)$ should be attained in $\bar{Q}_{T}$ on a parabolic boundary. Thus, 
$u(x, t)>0$ in $Q_{T}$, otherwise, there would be a contradiction with the initial datum. We show that $u(x, t)>0$ on $\partial \Omega \times(0, T)$. Let there exist a point $\left(x_{0}, t_{0}\right) \in S_{T}$ such that $u\left(x_{0}, t_{0}\right)=0$. But $u\left(x, t_{0}\right)>0$ for $x \in \Omega$. By boundary conditions (1) and assumption for $k_{1}(x, y, t)$ we have $u(x, t)>0$ for $(x, t) \in S_{T}$. This contradiction shows that $u(x, t)>0$ on $S_{T}$, and therefore $u(x, t)>0$ in $\bar{Q}_{T}$ for $0<t<T$.

Now we show the positiveness of $v(x, t)$. If $v_{0}(x)$ is a nontrivial function, then $v(x, t)>0$ in $\bar{Q}_{T}$ for $0<t<T$ by previous arguments. If $v_{0}(x) \equiv 0$ we suppose that there exists a constant $\tau>0$ such that $v(x, t) \equiv 0$ in $Q_{\tau}$ since otherwise we can use the arguments from the beginning of the proof again. But this is a contradiction with the second equation in (1) since $u(x, t)>0$ in $Q_{\tau}$ and $c_{2}(x, t)$ has the property $(N)$. Hence, we conclude that $v(x, t)>0$ in $\bar{Q}_{T}$ for $0<t<T$.

\section{Uniqueness and Nonuniqueness}

As a simple consequence of Theorem 4 and Theorem 9 we get the first uniqueness result for problem (1).

Theorem 10. Let problem (1) have a solution in $Q_{T}$ with nonnegative initial datum for $\min (p, q, m, n) \geq 1$ and with positive initial datum under conditions $\min (p, q, m, n)<1$ and $k_{1}(x, \cdot, t), k_{2}(x, \cdot, t)$ are nontrivial functions for any $x \in \partial \Omega$ and $t \in(0, T)$. Then solution of (1) is unique in $Q_{T}$.

Now we show nonuniqueness of solutions of problem (1) with trivial initial datum for $\min (p q, m, n)<1$.

Theorem 11. Let $\min (p q, m, n)<1, u_{0}(x)=v_{0}(x) \equiv 0$. Suppose that the maximal solution of problem (1) exists in $Q_{T}$. Assume that at least one of the following conditions is fulfilled:

$$
\begin{gathered}
c_{1}\left(x_{0}, t_{0}\right)>0, \quad c_{2}\left(x_{0}, t_{0}\right)>0 \\
\text { for some } x_{0} \in \Omega, t_{0} \in[0, T) \quad \text { if } p q<1, \\
k_{1}\left(x, y_{1}, t_{1}\right)>0
\end{gathered}
$$

for any $x \in \partial \Omega$, and some $y_{1} \in \partial \Omega, \quad t_{1} \in[0, T)$ if $m<1$,

$$
k_{2}\left(x, y_{2}, t_{2}\right)>0
$$

for any $x \in \partial \Omega$, and some $y_{2} \in \partial \Omega, \quad t_{2} \in[0, T)$ if $n<1$.

Then the maximal solution of problem (1) is nontrivial in $Q_{T}$.

Proof. In the local existence theorem we constructed a maximal solution $\left(u_{\max }(x, t), v_{\max }(x, t)\right)$ of (1) in the following way: $u_{\max }(x, t)=\lim _{\varepsilon \rightarrow 0} u_{\varepsilon}(x, t), v_{\max }(x, t)=\lim _{\varepsilon \rightarrow 0} v_{\varepsilon}(x, t)$, where $\left(u_{\varepsilon}(x, t), v_{\varepsilon}(x, t)\right)$ is some positive supersolution of $(1)$. To prove the theorem we construct a nontrivial nonnegative subsolution $(\underline{u}(x, t), \underline{v}(x, t))$ of some problem with trivial initial datum. By the comparison principle we conclude that $\left.u_{\varepsilon}(x, t) \geq \underline{u}(x, t), v_{\varepsilon}(x, t)\right) \geq \underline{v}(x, t)$, and therefore maximal solution is a nontrivial solution.

Consider at first the case when $p q<1$ and $c_{1}\left(x_{0}, t_{0}\right)>$ $0, c_{2}\left(x_{0}, t_{0}\right)>0$ for some $x_{0} \in \Omega$ and $0 \leq t_{0}<T$. Then there exists a neighborhood $U\left(x_{0}\right)$ of $x_{0}$ in $\Omega$ and $\bar{t} \in\left(t_{0}, T\right)$ such that $c_{1}(x, t) \geq c_{0}, c_{2}(x, t) \geq c_{0}, c_{0}>0$ for $x \in$ $U\left(x_{0}\right)$ and $t_{0} \leq t \leq \bar{t}$.

Consider the following problem:

$$
\begin{gathered}
w_{t}=\Delta w, \quad x \in U\left(x_{0}\right), t_{0}<t<\bar{t}, \\
w(x, t)=0, \quad x \in \partial U\left(x_{0}\right), t_{0}<t<\bar{t}, \\
w\left(x, t_{0}\right)=w_{0}(x), \quad x \in U\left(x_{0}\right),
\end{gathered}
$$

where $w_{0}(x)$ is a bounded nontrivial nonnegative continuous function which satisfies a boundary condition. By the strong maximum principle $0<w(x, t)<M_{0}$ for $t \in\left(t_{0}, \bar{t}\right), x \in$ $U\left(x_{0}\right)$, where $M_{0}=\sup _{x \in U\left(x_{0}\right)} w_{0}(x)$.

Let $\alpha=(1+p) /(1-p q)>1, \beta=(1+q)(1-p q)>1$. Denote that

$$
\begin{aligned}
& \underline{u}(x, t)=C^{\alpha}\left(t-t_{0}\right)^{\alpha} w^{\alpha}(x, t), \\
& \underline{v}(x, t)=C^{\beta}\left(t-t_{0}\right)^{\beta} w^{\beta}(x, t),
\end{aligned}
$$

where $C=\min \left(c_{0} /\left(\alpha M_{0}\right), c_{0} /\left(\beta M_{0}\right)\right)$. Note that $\underline{u}(x, t)=0$, $\underline{v}(x, t)=0$ if $t=t_{0}$ or $x \in \partial U\left(x_{0}\right)$. After simple calculations we obtain

$$
\begin{aligned}
\underline{u}_{t}- & \Delta \underline{u}-c_{1}(x, t) \underline{v}^{p}=\alpha C^{\alpha}\left(t-t_{0}\right)^{\alpha-1} w^{\alpha} \\
& +\alpha C^{\alpha}\left(t-t_{0}\right)^{\alpha} w^{\alpha-1} w_{t}-\alpha \Delta w w^{\alpha-1} C^{\alpha}\left(t-t_{0}\right)^{\alpha} \\
& -\alpha(\alpha-1) w^{\alpha-2}|\nabla w|^{2} C^{\alpha}\left(t-t_{0}\right)^{\alpha} \\
& -c_{1}(x, t) C^{\beta p}\left(t-t_{0}\right)^{\beta p} w^{\beta p} \\
\leq & C^{\beta p}\left(t-t_{0}\right)^{\beta p} w^{\beta p}\left(\alpha C w-c_{1}(x, t)\right) \leq 0 .
\end{aligned}
$$

Similarly we can get that

$$
\underline{v}_{t}-\Delta \underline{v}-c_{2}(x, t) \underline{u}^{q} \leq 0 .
$$

Then $(\underline{u}(x, t), \underline{v}(x, t))$ and $\left(u_{\varepsilon}(x, t), v_{\varepsilon}(x, t)\right)$ are subsolution and supersolution, respectively, of the following problem:

$$
\begin{aligned}
& u_{t}=\Delta u+c_{1}(x, t) v^{p}, \\
& v_{t}=\Delta v+c_{2}(x, t) u^{q}, \\
& x \in U\left(x_{0}\right), t_{0}<t<\bar{t}, \\
& u(x, t)=v(x, t)=0, \\
& x \in \partial U\left(x_{0}\right), t_{0}<t<\bar{t}, \\
& u\left(x, t_{0}\right)=v\left(x, t_{0}\right)=0, \\
& x \in U\left(x_{0}\right) .
\end{aligned}
$$

By comparison principle for (38) we conclude that $u_{\varepsilon}(x, t) \geq$ $\underline{u}(x, t), v_{\varepsilon}(x, t) \geq \underline{v}(x, t)$ and hence $u_{\max }(x, t) \geq \underline{u}(x, t)$, $v_{\max }(x, t) \geq \underline{v}(x, t)$, for $(x, t) \in U\left(x_{0}\right) \times\left(t_{0}, \bar{t}\right)$. 
Now consider the case when $m<1$ and $k_{1}\left(x, y_{1}, t_{1}\right)>0$ for any $x \in \partial \Omega$ and some $y_{1} \in \partial \Omega, 0 \leq t_{1}<T$. We will consider the following problem:

$$
\begin{gathered}
u_{t}=\Delta u+c_{1}(x, t) v^{p}, \\
v_{t}=\Delta v+c_{2}(x, t) u^{q}, \\
x \in \Omega, t_{1}<t<T_{0}, \\
u(x, t)=\int_{\Omega} k_{1}(x, y, t) u^{m}(y, t) d y, \\
x \in \partial \Omega, t_{1}<t<T_{0}, \\
v(x, t)=0, \quad x \in \partial \Omega, t_{1}<t<T_{0}, \\
u\left(x, t_{1}\right)=0, \quad v\left(x, t_{1}\right)=0, \quad x \in \Omega,
\end{gathered}
$$

where $T_{0}>t_{1}$ will be defined later. Construct a subsolution of (39) using the change of variables in a neighborhood of $\partial \Omega$ as in [23]. Let $\bar{x} \in \partial \Omega$. We denote by $\widehat{n}$ the inner unit normal to $\partial \Omega$ at the point $\bar{x}$. Since $\partial \Omega$ is smooth it is well known that there exists $\delta>0$ such that the mapping $\psi: \partial \Omega \times[0, \delta] \rightarrow$ $\mathbb{R}^{N}$ given by $\psi(\bar{x}, s)=\bar{x}+s \widehat{n}(\bar{x})$ defines new coordinates $(\bar{x}, s)$ in a neighborhood of $\partial \Omega$ in $\bar{\Omega}$. A straightforward computation shows that, in these coordinates, $\Delta$ applied to a function $g(\bar{x}, s)=g(s)$ which is independent of the variable $\bar{x}$, evaluated at a point $(\bar{x}, s)$ is given by

$$
\Delta g(\bar{x}, s)=\frac{\partial^{2} g}{\partial s^{2}}(\bar{x}, s)-\sum_{j=1}^{N-1} \frac{H_{j}(\bar{x})}{1-s H_{j}(\bar{x})} \frac{\partial g}{\partial s}(\bar{x}, s)
$$

where $H_{j}(\bar{x}), j=1, \ldots, N-1$ denote the principal curvatures of $\partial \Omega$ at $\bar{x}$.

Under the made assumption there exists $\tilde{t}>0$ such that $k_{1}(x, y, t)>0$ for $t_{1} \leq t \leq t_{1}+\tilde{t}, x \in \partial \Omega, y \in V\left(y_{1}\right)$, where $V\left(y_{1}\right)$ is some neighborhood of $y_{1}$ in $\bar{\Omega}$. Let $\alpha>1 /(2(1-m))$ and assume that $0<\xi_{0} \leq 1$ and $t_{1}<T_{0} \leq t_{1}+\min \left(\tilde{t}, \delta^{2}, T-\right.$ $\left.t_{1}\right)$. For points in $\partial \Omega \times[0, \delta] \times\left(t_{1}, T_{0}\right]$ of coordinates $(\bar{x}, s, t)$ define

$$
\begin{gathered}
\underline{u}(\bar{x}, s, t)=\left(t-t_{1}\right)^{\alpha}\left(\xi_{0}-\frac{s}{\sqrt{t-t_{1}}}\right)_{+}^{3}, \\
\underline{v}(\bar{x}, s, t)=0
\end{gathered}
$$

and extend $\underline{u}, \underline{v}$ as zero to the whole $\bar{\Omega} \times\left[t_{1}, T_{0}\right]$. Using (40), we get that

$$
\begin{gathered}
\underline{u}_{t}(\bar{x}, s, t)-\Delta \underline{u}(\bar{x}, s, t)-c_{1}(x, t) \underline{v}^{p}(\bar{x}, s, t) \\
=\frac{3 s\left(t-t_{1}\right)^{\alpha-3 / 2}}{2}\left(\xi_{0}-\frac{s}{\sqrt{t-t_{1}}}\right)_{+}^{2} \\
+\alpha\left(t-t_{1}\right)^{\alpha-1}\left(\xi_{0}-\frac{s}{\sqrt{t-t_{1}}}\right)_{+}^{3}
\end{gathered}
$$

$$
\begin{aligned}
& -6\left(t-t_{1}\right)^{\alpha-1}\left(\xi_{0}-\frac{s}{\sqrt{t-t_{1}}}\right)_{+} \\
& -3\left(t-t_{1}\right)^{\alpha-1 / 2}\left(\xi_{0}-\frac{s}{\sqrt{t-t_{1}}}\right)_{+}^{2} \\
& \times \sum_{j=1}^{N-1} \frac{H_{j}(\bar{x})}{1-s H_{j}(\bar{x})} \leq 0
\end{aligned}
$$

for sufficiently small values of $\xi_{0}$. It is clear that

$$
\underline{v}_{t}(\bar{x}, s, t)-\Delta \underline{v}(\bar{x}, s, t)-c_{2}(x, t) \underline{u}^{q}(\bar{x}, s, t) \leq 0 .
$$

It remains to verify the validity of the inequality

$$
\begin{aligned}
\left(t-t_{1}\right)^{\alpha} \xi_{0}^{3} \leq & \left(t-t_{1}\right)^{\alpha m} \\
& \times \int_{\partial \Omega \times[0, \delta]} k_{1}(x,(\bar{y}, s), t)|J(\bar{y}, s)| \\
& \times\left(\xi_{0}-\frac{s}{\sqrt{t-t_{1}}}\right)_{+}^{3 m} d \bar{y} d s
\end{aligned}
$$

for $x \in \partial \Omega$ and $t_{1}<t<T_{0}$. Here $J(\bar{y}, s)$ is Jacobian of the change of variables. Estimating the integral I in the righthand side of (44) we get

$$
\begin{aligned}
& I=(\left.t-t_{1}\right)^{1 / 2} \\
& \times \int_{\partial \Omega} d \bar{y} \int_{0}^{\xi_{0}} k_{1}\left(x,\left(\bar{y}, z \sqrt{t-t_{1}}\right), t\right) \\
& \times\left|J\left(\bar{y}, z \sqrt{t-t_{1}}\right)\right|\left(\xi_{0}-z\right)_{+}^{3 m} d z \\
& \geq C\left(t-t_{1}\right)^{1 / 2}
\end{aligned}
$$

where constant $C$ does not depend on $t$; we obtain that (44) is true if $T_{0}-t_{1}$ is small enough.

By comparison principle for (39) we conclude that $u_{\varepsilon}(x, t) \geq \underline{u}(x, t), v_{\varepsilon}(x, t) \geq \underline{v}(x, t)$ and, respectively, $u_{\max }(x, t) \geq \underline{u}(x, t), v_{\max }(x, t) \geq \underline{v}(x, t)$ for $(x, t) \in \Omega \times$ $\left(t_{1}, T_{0}\right)$.

The proof in the case $n<1$ and $k_{2}\left(x, y_{2}, t_{2}\right)>0$ for any $x \in \partial \Omega$ and some $y_{2} \in \partial \Omega, 0 \leq t_{2}<T$ is similar.

It is easy to get from Theorem 9 and the proof of Theorem 11 the following statement.

Corollary 12. Let the conditions of Theorem 11 hold with $t_{i}=$ $0, i=0,1,2$. Suppose also that $k_{1}(x, \cdot, t)$ and $k_{2}(x, \cdot, t)$ are nontrivial functions for any $x \in \partial \Omega$ and $t \in(0, T), c_{2}(x, t)$ has the property $(N)$ if $(32)$ is realized, and $c_{1}(x, t)$ has the property $(N)$ if (33) is realized. Then maximal solution is positive in $\bar{Q}_{T}$ for $0<t<T$.

Under the conditions of Corollary 12 for some class of the coefficients $c_{i}(x, t)$ and $k_{i}(x, y, t), i=1,2$, we can prove the uniqueness of solution for (1) with trivial initial datum which is positive for all positive times as long as it exists. 
Theorem 13. Let the conditions of Corollary 12 hold. Suppose also that there exists $t_{0}>0$ such that for $0 \leq t \leq t_{0}$ the functions $c_{1}(x, t), c_{2}(x, t), k_{1}(x, y, t)$, and $k_{2}(x, y, t)$ are nondecreasing with respect to $t$.

Then there exists exactly one solution of problem (1) which is positive in $\bar{Q}_{T}$ for $0<t<T$.

Proof. Suppose that there exists different from $\left(u_{\max }(x, t)\right.$, $\left.v_{\max }(x, t)\right)$ solution $(u(x, t), v(x, t))$ of (1) with trivial initial datum which is a positive in $\bar{Q}_{T}$ for $0<t<T$. Denote $t_{*}=\min \left(t_{0}, T\right)$. Due to the conditions of the theorem it is easy to see that $u(x, t+\tau), v(x, t+\tau)$ is positive supersolution of (1) with trivial initial datum in $Q_{t_{*}-\tau}$ for any $\tau \in\left(0, t_{*}\right)$. By Theorem 4 we have $u_{\text {max }}(x, t) \leq u(x, t+\tau), v_{\max }(x, t) \leq$ $v(x, t+\tau)$ for every $0 \leq t \leq t_{*}-\tau$. Passing to the limit as $\tau \rightarrow 0$ we get $u_{\max }(x, t) \leq u(x, t), v_{\max }(x, t) \leq v(x, t)$ for $0 \leq t \leq t_{*}$. Hence $u_{\max }(x, t)=u(x, t), v_{\max }(x, t)=v(x, t)$ in $Q_{T}$.

Note that by Theorem 10 , the solution $(u(x, t), v(x, t))$ of (1) is unique if $\min (p, q, m, n) \geq 1$. Now we specify our uniqueness result in the case $\min (p q, m, n)<1$.

Theorem 14. Let the conditions of Corollary 12 fulfill only $u_{0}(x) \neq \equiv$ or $v_{0}(x) \neq \equiv 0$. Then the solution of $(1)$ is unique.

Proof. To prove the uniqueness of the solution if $\min (p q$, $m, n)<1$, it suffices to show that if $(u(x, t), v(x, t))$ is any solution of (1), then

$$
u_{\max }(x, t) \leq u(x, t), \quad v_{\max }(x, t) \leq v(x, t),
$$

where $\left(u_{\max }(x, t), v_{\max }(x, t)\right)$ is a maximal solution of $(1)$.

First, consider the case when $0<p<1,0<q \leq 1,0<$ $m<1$, and $0<n \leq 1$. Let

$$
\begin{aligned}
& w_{1}(x, t)=u_{\max }(x, t)-u(x, t), \\
& w_{2}(x, t)=v_{\max }(x, t)-v(x, t) .
\end{aligned}
$$

Then $\left(w_{1}, w_{2}\right)$ satisfies

$$
\begin{gathered}
w_{1 t} \leq \Delta w_{1}+c_{1}(x, t) w_{2}^{p}, \\
w_{2 t} \leq \Delta w_{2}+c_{2}(x, t) w_{1}^{q}, \\
x \in \Omega, \quad 0<t<T_{1}, \\
w_{1}(x, t) \leq \int_{\Omega} k_{1}(x, y, t) w_{1}^{m}(y, t) d y, \\
w_{2}(x, t) \leq \int_{\Omega} k_{2}(x, y, t) w_{2}^{n}(y, t) d y, \quad 0<t<T_{1}, \\
x \in \partial \Omega, \quad 0<t<T_{1}, \\
w_{1}(x, 0) \equiv 0, \quad w_{2}(x, 0) \equiv 0, \quad x \in \Omega
\end{gathered}
$$

for some $T_{1}>0$. By Theorem 13, there exists a unique solution $\left(h_{1}(x, t), h_{2}(x, t)\right)$ of the following problem:

$$
\begin{gathered}
h_{1 t}=\Delta h_{1}+c_{1}(x, t) h_{2}^{p}, \\
h_{2 t}=\Delta h_{2}+c_{2}(x, t) h_{1}^{q}, \\
x \in \Omega, \quad 0<t<T_{2}, \\
h_{1}(x, t)=\int_{\Omega} k_{1}(x, y, t) h_{1}^{m}(y, t) d y, \\
h_{2}(x, t)=\int_{\Omega} k_{2}(x, y, t) h_{2}^{n}(y, t) d y, \\
x \in \partial \Omega, \quad 0<t<T_{2}, \\
h_{1}(x, 0) \equiv 0, \quad h_{2}(x, 0) \equiv 0, \quad x \in \Omega,
\end{gathered}
$$

such that $h_{1}(x, t)>0, h_{2}(x, t)>0$ for $x \in \bar{\Omega}, 0<t<T_{2}$ for some $T_{2}>0$. Let $T_{0}=\min \left(T_{1}, T_{2}\right)$. In a similar way as in Theorems 13 and 4 we can prove that $u_{\max }(x, t) \geq h_{1}(x, t)$ $\geq w_{1}(x, t), v_{\max }(x, t) \geq h_{2}(x, t) \geq w_{2}(x, t)$. Put $a_{1}=h_{1}-$ $w_{1}, a_{2}=h_{2}-w_{2}$. Now, use an elementary inequality, which is recalled for instance in [20],

$$
a^{k}-b^{k}+c^{k} \geq(a-b+c)^{k},
$$

where $0<k \leq 1, \min (a, b, c)>0$, and $\max (a, c) \leq b \leq a+c$. Then we obtain

$$
\begin{aligned}
& a_{1 t} \geq \Delta a_{1}+c_{1}(x, t) a_{2}^{p}, \quad a_{2 t} \geq \Delta a_{2}+c_{2}(x, t) a_{1}^{q}, \\
& x \in \Omega, \quad 0<t<T_{0}, \\
& a_{1}(x, t) \geq \int_{\Omega} k_{1}(x, y, t) a_{1}^{m}(y, t) d y, \\
& x \in \partial \Omega, \quad 0<t<T_{0}, \\
& a_{2}(x, t) \geq \int_{\Omega} k_{2}(x, y, t) a_{2}^{n}(y, t) d y, \\
& x \in \partial \Omega, \quad 0<t<T_{0}, \\
& a_{1}(x, 0) \equiv 0, \quad a_{2}(x, 0) \equiv 0, \quad x \in \Omega \text {. }
\end{aligned}
$$

We show that $a_{1}(x, t)>0, a_{2}(x, t)>0$ in $Q_{T_{0}}$. In fact, otherwise, by Theorem 9 there exists $\bar{t} \in\left(0, T_{0}\right)$ such that $a_{1}(x, t)=a_{2}(x, t) \equiv 0$ in $Q_{\bar{t}}$. Suppose that $c_{1}\left(x_{0}, 0\right)>0$ for some $x_{0} \in \Omega$. Then we get

$$
\begin{aligned}
c_{1}\left(h_{2}+v\right)^{p} & =c_{1}\left(w_{2}+v\right)^{p}=c_{1} v_{\max }^{p} \\
& =\left(u_{\max }\right)_{t}-\Delta u_{\max }=\left(w_{1}+u\right)_{t}-\Delta\left(w_{1}+u\right) \\
& =\left(h_{1}+u\right)_{t}-\Delta\left(h_{1}+u\right)=c_{1}\left(h_{2}^{p}+v^{p}\right)
\end{aligned}
$$

for $x \in \Omega$ and $0<t<\bar{t}$. This is a contradiction since $h_{2}(x, t)>$ $0, v(x, t)>0$ in $Q_{T_{0}}, c_{1}\left(x_{0}, t_{0}\right)>0$ for some $t_{0} \in(0, \bar{t})$, and $0<p<1$. 
If $k_{1}\left(x, y_{0}, 0\right)>0$ for any $x \in \partial \Omega$ and some $y_{0} \in \partial \Omega$ we can obtain a contradiction by another way. Indeed,

$$
\begin{aligned}
& \int_{\Omega} k_{1}(x, y, t)\left(h_{1}^{m}(y, t)+u^{m}(y, t)\right) d y \\
& =h_{1}(x, t)+u(x, t)=w_{1}(x, t)+u(x, t) \\
& =u_{\max }(x, t)=\int_{\Omega} k_{1}(x, y, t) u_{\max }^{m}(y, t) d y \\
& =\int_{\Omega} k_{1}(x, y, t)\left(w_{1}(y, t)+u(y, t)\right)^{m} d y \\
& =\int_{\Omega} k_{1}(x, y, t)\left(h_{1}(y, t)+u(y, t)\right)^{m} d y
\end{aligned}
$$

for $x \in \partial \Omega$ and $0<t<\bar{t}$ and we get again a contradiction since $h_{1}(x, t)>0, u(x, t)>0$ in $Q_{T_{0}}$, and $0<m<1$.

Since $a_{1}>0, a_{2}>0$ in $Q_{T_{0}}$ by comparison principle with arguments of Theorems 13 and 4 we conclude that $a_{1} \geq$ $h_{1}, a_{2} \geq h_{2}$ in $Q_{T_{0}}$. This implies (46) and completes the proof for the first case.

Now suppose that $\max (p, q, m, n)>1$. Assume, for example, that $0<p \leq 1, q>1,0<m<1, n>1$. Then, as in the first case, we introduce the functions $w_{1}(x, t), w_{2}(x, t)$. We use the following relations:

$$
\begin{aligned}
u_{\max }^{q}(x, t)-u^{q}(x, t) & =q \theta_{1}^{q-1}\left(u_{\max }(x, t)-u(x, t)\right) \\
& \leq M_{1}\left(u_{\max }(x, t)-u(x, t)\right), \\
v_{\max }^{n}(x, t)-v^{n}(x, t) & =n \theta_{2}^{n-1}\left(v_{\max }(x, t)-v(x, t)\right) \\
& \leq M_{2}\left(v_{\max }(x, t)-v(x, t)\right),
\end{aligned}
$$

where $\theta_{1}, \theta_{2}$ are nonnegative continuous functions which are between $u_{\max }(x, t), u(x, t)$ and $v_{\max }(x, t), v(x, t)$, respectively, $q \theta_{1}^{q-1} \leq M_{1}, n \theta_{2}^{n-1} \leq M_{2}$ in $Q_{T}$. Then we have that a pair of functions $\left(w_{1}(x, t), w_{2}(x, t)\right)$ satisfies relations

$$
\begin{gathered}
w_{1 t} \leq \Delta w_{1}+c_{1}(x, t) w_{2}^{p}, \\
w_{2 t} \leq \Delta w_{2}+c_{2}(x, t) M_{1} w_{1}, \\
x \in \Omega, \quad 0<t<T_{1}, \\
w_{1}(x, t) \leq \int_{\Omega} k_{1}(x, y, t) w_{1}^{m}(y, t) d y, \\
w_{2}(x, t) \leq \int_{\Omega} k_{2}(x, y, t) M_{2} w_{2}(y, t) d y, \quad 0<t<T_{1}, \\
w_{1}(x, 0) \equiv 0, \quad w_{2}(x, 0) \equiv 0, \quad x \in \Omega .
\end{gathered}
$$

Further develop the arguments, as in the first case, only for $q=1, n=1$. Using the linearization of terms with powers greater than 1 in the equations and boundary conditions of (1) as above we can prove the theorem for the remaining cases in a similar way.

\section{Conflict of Interests}

The authors declare that there is no conflict of interests regarding the publication of this paper.

\section{Acknowledgments}

The authors would like to thank the referees for their valuable comments and suggestions regarding the original paper.

\section{References}

[1] B. Chen, Y. Mi, and C. Mu, "A quasilinear parabolic system with nonlocal boundary condition," Boundary Value Problems, vol. 2011, Article ID 750769, 2011.

[2] Z. Cui and Z. Yang, "Roles of weight functions to a nonlinear porous medium equation with nonlocal source and nonlocal boundary condition," Journal of Mathematical Analysis and Applications, vol. 342, no. 1, pp. 559-570, 2008.

[3] K. Deng, "Comparison principle for some nonlocal problems," Quarterly of Applied Mathematics, vol. 50, no. 3, pp. 517-522, 1992.

[4] A. Friedman, "Monotone decay of solutions of parabolic equations with nonlocal boundary conditions," Quarterly of Applied Mathematics, vol. 44, no. 3, pp. 401-407, 1986.

[5] A. Gladkov and M. Guedda, "Blow-up problem for semilinear heat equation with absorption and a nonlocal boundary condition," Nonlinear Analysis: Theory, Methods \& Applications, vol. 74, no. 13, pp. 4573-4580, 2011.

[6] A. Gladkov and K. I. Kim, "Blow-up of solutions for semilinear heat equation with nonlinear nonlocal boundary condition," Journal of Mathematical Analysis and Applications, vol. 338, no. 1, pp. 264-273, 2008.

[7] D. Liu and C. Mu, "Blowup properties for a semilinear reactiondiffusion system with nonlinear nonlocal boundary conditions," Abstract and Applied Analysis, vol. 2010, Article ID 148035, 17 pages, 2010.

[8] D. Liu, C. Mu, and I. Ahmed, "Blow-up for a semilinear parabolic equation with nonlinear memory and nonlocal nonlinear boundary," Taiwanese Journal of Mathematics, vol. 17, no. 4, pp. 1353-1370, 2013.

[9] P. Souplet, "Uniform blow-Up profiles and boundary behavior for diffusion equations with nonlocal nonlinear source," Journal of Differential Equations, vol. 153, no. 2, pp. 374-406, 1999.

[10] Z. Ye and X. Xu, "Global existence and blow-up for a porous medium system with nonlocal boundary conditions and nonlocal sources," Nonlinear Analysis: Theory, Methods \& Applications, vol. 82, pp. 115-126, 2013.

[11] Y. F. Yin, "On nonlinear parabolic equations with nonlocal boundary condition," Journal of Mathematical Analysis and Applications, vol. 185, no. 1, pp. 161-174, 1994.

[12] S. Zheng and L. Kong, "Roles of weight functions in a nonlinear nonlocal parabolic system," Nonlinear Analysis: Theory, Methods \& Applications, vol. 68, no. 8, pp. 2406-2416, 2008.

[13] C. Cortazar, M. Elqueta, and J. D. Rossi, "Uniqueness and nonuniqueness for the porous medium equation with nonlinear boundary condition," Differential and Integral Equations, vol. 16, no. 10 , pp. 1215-1222, 2003.

[14] H. Fujita and S. Watanabe, "On the uniqueness and nonuniqueness of solutions of initial value problems for some 
quasi-linear parabolic equations," Communications on Pure and Applied Mathematics, vol. 21, no. 6, pp. 631-652, 1968.

[15] A. Gladkov and M. Guedda, "Semilinear heat equation with absorption and a nonlocal boundary condition," Applicable Analysis, vol. 91, no. 12, pp. 2267-2276, 2012.

[16] A. Gladkov and K. I. Kim, "Uniqueness and nonuniqueness for reaction-diffusion equation with nonlocal boundary condition," Advances in Mathematical Sciences and Applications, vol. 19, pp. 39-49, 2009.

[17] P. Bokes, "A uniqueness result for a semilinear parabolic system," Journal of Mathematical Analysis and Applications, vol. 331, no. 1, pp. 567-584, 2007.

[18] C. Cortazar, M. Elgueta, and J. D. Rossi, "Uniqueness and non-uniqueness for a system of heat equations with nonlinear coupling at the boundary," Nonlinear Analysis: Theory, Methods \& Applications, vol. 37, no. 2, pp. 257-267, 1999.

[19] F. Dickstein and M. Escobedo, "A maximum principle for semilinear parabolic systems and applications," Nonlinear Analysis: Theory, Methods \& Applications, vol. 45, no. 7, pp. 825-837, 2001.

[20] M. Escobedo and M. A. Herrero, "A semilinear parabolic system in a bounded domain," Annali di Matematica Pura ed Applicata, vol. 165, no. 1, pp. 315-336, 1993.

[21] M. Kordoš, "On uniqueness for a semilinear parabolic system coupled in an equation and a boundary condition," Journal of Mathematical Analysis and Applications, vol. 298, no. 2, pp. 655666, 2004.

[22] O. A. Ladyzhenskaja, V. A. Solonnikov, and N. N. Ural'ceva, Linear and Quasilinear Equations of Parabolic Type, vol. 23 of Translations of Mathematical Monographs, American Mathematical Society, Providence, RI, USA, 1968.

[23] C. Cortázar, M. del Pino, and M. Elgueta, "On the short-time behavior of the free boundary of a porous medium equation," Duke Mathematical Journal, vol. 87, no. 1, pp. 133-149, 1997. 


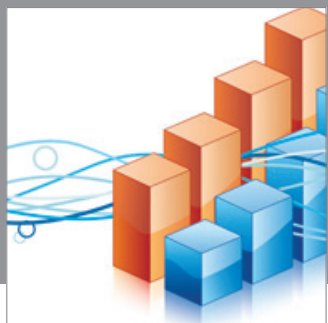

Advances in

Operations Research

mansans

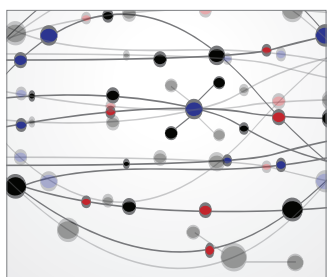

The Scientific World Journal
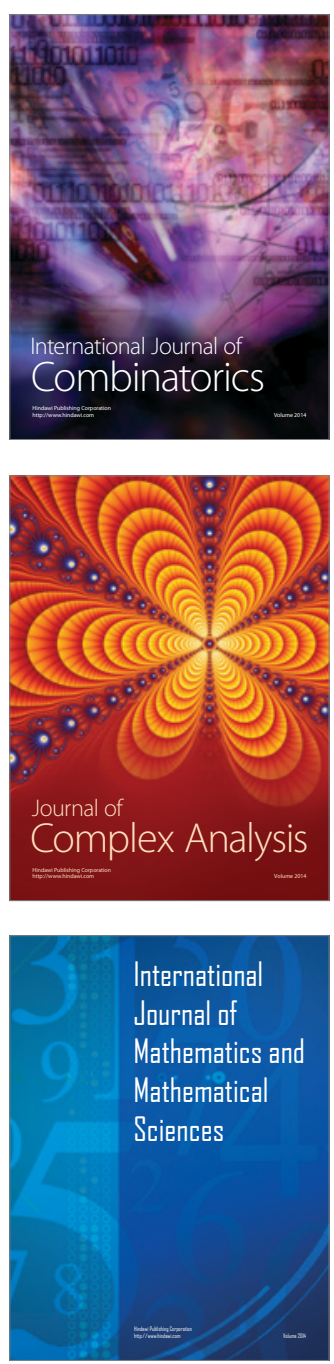
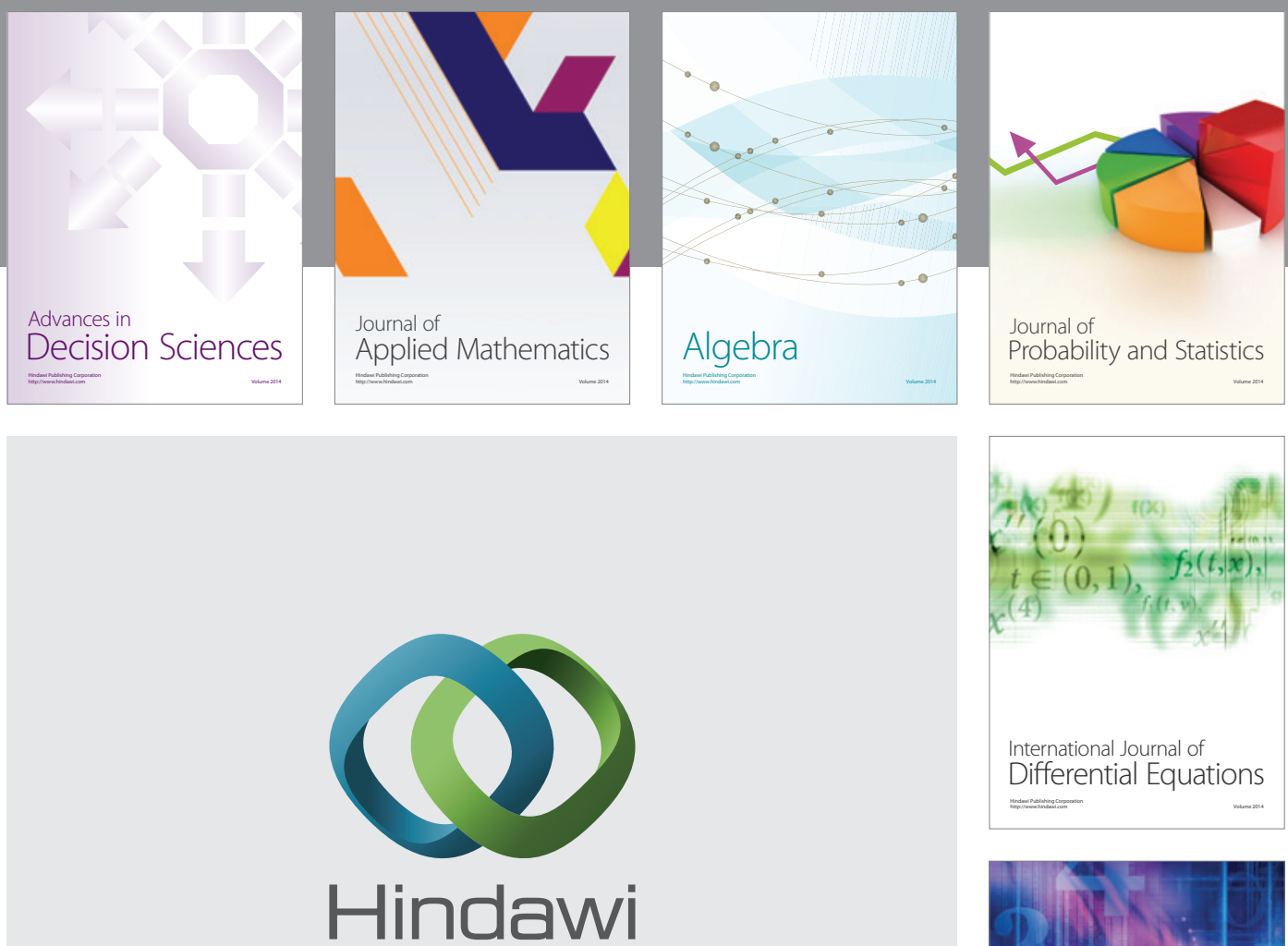

Submit your manuscripts at http://www.hindawi.com
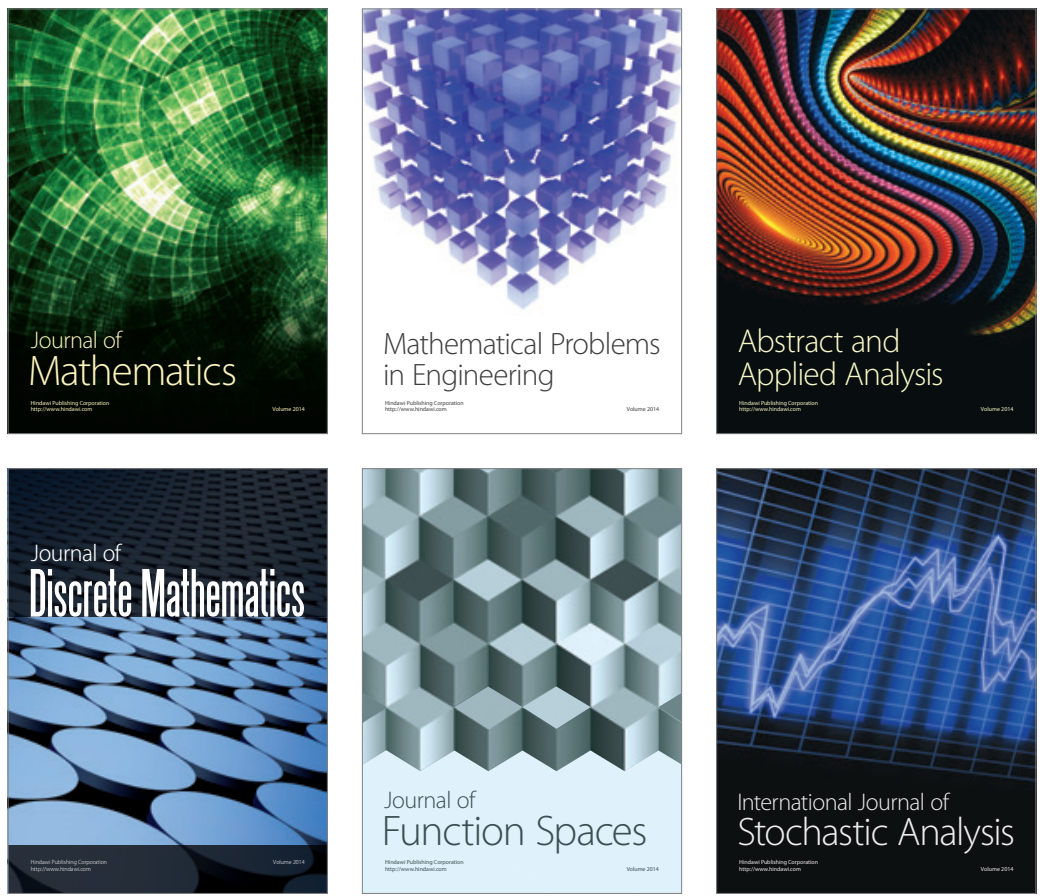

Journal of

Function Spaces

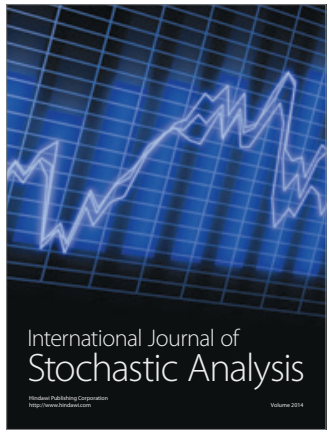

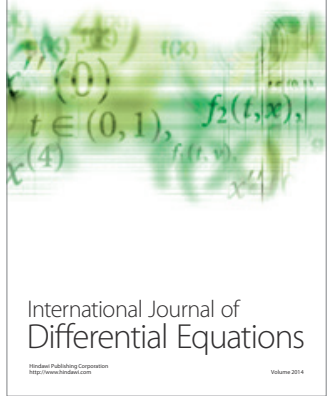
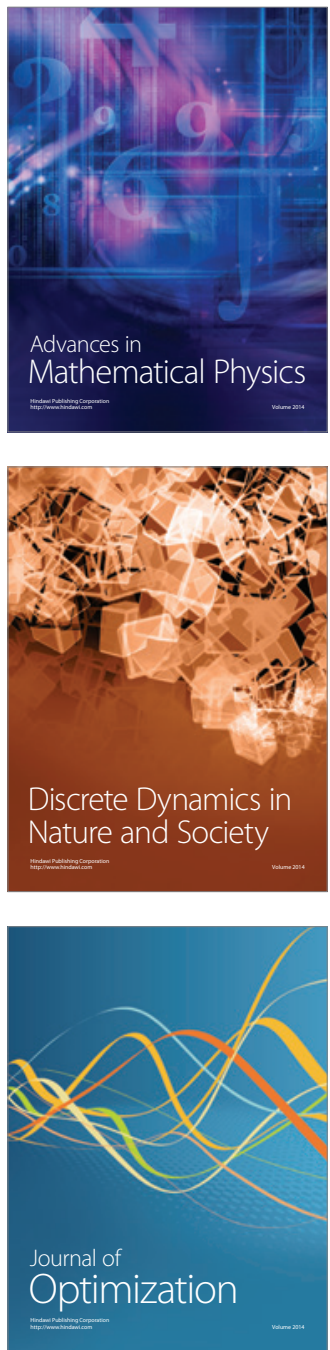